\title{
音叉式引力波天线的隔振 及空气弹簧的研究
}

赵志强阎永廉秦荣

(中国科学院高能物理研究所, 北京)

音叉式引力波天线 $[1,2]$ 是用来探测脉冲星低频连续引力波的谐振型天线. 目前, 高能物理 研究所引力室正在研制用于探测 Vela 脉冲星引力波的集中质量音叉天线, 其谐振频率为 22.4 $\mathrm{Hz}$. 由于谐振频率较低, 而且地面振动的低频成分较强, 所以这种天线的隔振问题就更为突 出.

\section{一、音叉天线对隔振的要求}

由于连续引力波探测与爆发引力波探测的原理有所不同, 所以对隔振的要求也不同. 尤 其音叉天线,由于采用信号同步积累的检测方法，对各种噪声干扰的抑制能力可大为加强 $[1,3]$. 我们认为, 音叉天线对隔振的要求是, 使外界机械噪声被衰减到天线热噪声的水平. 从这一观 点出发, 根据地面振动噪声的实测水平, 则可以算出所需要的隔振传输比.

我们对本实验室内的地面振动做了简单的测试. 所测得的地面振动位移谱密度在天线谐 振频率附近的值约为 $x_{s}^{2}=2 \times 10^{-17}(\mathrm{~m} / \sqrt{\mathrm{Hz}})^{2}$. 天线热躁声谱密度在天线谐振频率处的 值为 ${ }^{[4]}$

$$
x_{T}^{2}\left(\nu_{0}\right)=\frac{4 Q k T_{a n}}{M \omega_{0}^{3}},
$$

其中 $Q$ 为天线品质因数, $k$ 为 Boltzmann 常数, $T_{a n}$ 为天线温度, $M$ 为天线质量, $\omega_{0}=2 \pi \nu_{0}$ 为 天线谐振频率.

音叉天线对支点水平方向振动的响应很小，所以隔振主要考虑对垂直方向振动的衰减. 计算可得, 音叉天线通过其弹性支承对垂直方向外界振动的响应为

$$
\xi=2 \alpha_{0} Q\left(\frac{\omega_{3}}{\omega_{0}}\right)^{2} x_{z},
$$

其中 $x_{z}$ 为外界垂直振动幅度, $\xi$ 为音叉天线受检测面的相对运动幅度， $a_{0}$ 为天线结构参数,一 般取 $\alpha_{0} \sim 10^{-4}, \omega_{3}$ 为天线弹性支承的固有频率. 由(2)式可得天线通过其隔振系统对地面噪 声的响应为

$$
x_{s 0}^{2}\left(\nu_{0}\right)=4 \alpha_{0}^{2} Q^{2}\left|H\left(\nu_{0}\right)\right|^{2} x_{s}^{2}\left(\nu_{0}\right),
$$

其中 $x_{50}^{2}$ 为天线机械噪声谱密度, $H$ 为隔振系统的传输函数.

如前所述, 令 $x_{s 0}^{2}<x_{T}^{2}$, 则由 (1)、(3)式得

$$
\left|H\left(\nu_{0}\right)\right|<\frac{1}{\alpha_{0}}\left[\frac{k T_{a n}}{Q M \omega_{0}^{3}}\right]^{\frac{3}{2}}-\frac{1}{\sqrt{x_{s}^{2}\left(\nu_{0}\right)}} .
$$

本文 1985 年 11 月 25 日收到. 
对于 $200 \mathrm{~kg}$ 的天线 ${ }^{[1]}, M=200 \mathrm{~kg}, Q=4 \times 10^{7}, \alpha_{0}=10^{-4}, T_{a n}=4.2 \mathrm{~K}, \omega_{0}=2 \pi \times 22.4 \mathrm{~s}^{-1}$, $\sqrt{x_{s}^{2}}=4.5 \times 10^{-9} \mathrm{~m} / \sqrt{\mathrm{Hz}}$, 由(4)式得到 $\left|H\left(\nu_{0}\right)\right|<1.1 \times 10^{-7}(-139 \mathrm{~dB})$.

\section{二、隔振的具体方案}

我们准备采用 4 级隔振,包括: 空气弹簧隔振台, 采用自振频率小于 $1 \mathrm{~Hz}$ 的气缸-薄膜式 空气弹簧, 对 $22.4 \mathrm{~Hz}$ 振动的传输比可达 $-50 \mathrm{~dB}$. 在空气弹簧隔振台与低温容器之间, 采用一 级钢弹簧悬吊. 为了获得较低的频率和较好的稳定性, 准备采用拉伸螺旋弹簧. 若自振频率 为 $1.5 \mathrm{~Hz}$, 可达 $-47 \mathrm{~dB}$ 的传输比. 在低温容器内准备采用多层铝合金片 (悬臂梁和简支梁) 支 承, 构成两级隔振, 其传输比可达 $-68 \mathrm{~dB}$. 各级加起来总传输比为 $-165 \mathrm{~dB}$, 可以满足音义天 线对隔振的要求.

\section{三、空气弹簧的实验研究}

为了使空气弹簧隔振台达到较低的自振频率, 我们发展了一种气缸一薄膜式空气弹簧, 并 对其主要参数的合理选取进行了一些实验研究. 这种空气 弹簧的主要结构是气缸、活塞和橡胶薄膜,如图 1 所示. 由 气体多方过程方程可以导出空气弹簧的刚度表达式（当处 于平衡位置 $x=0$ 时) ${ }^{[5]}$ :

$$
k_{0}=r\left(p_{0}+p_{a}\right) \frac{A_{0}^{2}}{V_{0}}+\left.p_{0} \frac{d A}{d x}\right|_{x=0},
$$

式中 $\gamma$ 为多方指数, $1<\gamma<1.4$,一般取 $\gamma=1.4$; $p_{0}$ 为平 衡时气畋内气体压力 (表压力); $p_{a}$ 为大气压力; $A$ 为有效 面积, $A_{0}$ 为 $A$ 在平衡位置的值; $V_{0}$ 为气缸容积; $x$ 为活塞离 平衡位置的位移.

我们在自制的空气弹簧上, 在载荷 $W=p_{0} A_{0}$ 不变的 条件下,取不同的 $A_{0}$ 和橡胶膜的不同䆓度和厚度进行了一 系列的试验, 以便找到合理的参数取值, 降低自振频率. 在 实验中发现, 当刚度 $k_{0}$ 较小时, 不能忽略橡胶膜本身非柔

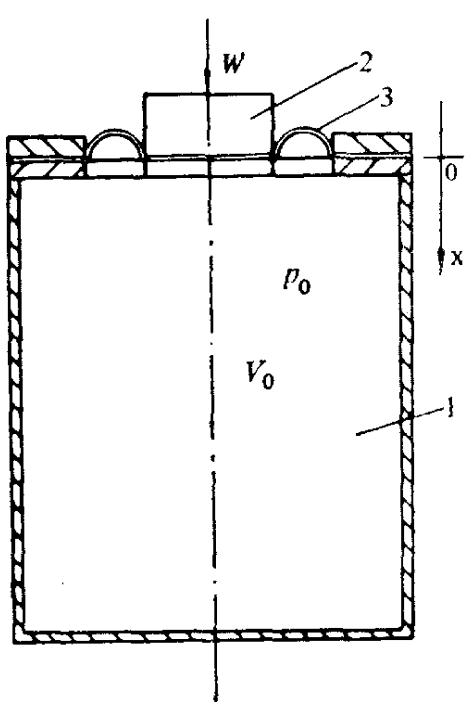

图 1

1 .气征； 2 .活骞； 3 .侬胶薄膜 软性对刚度的贡献. 我们以 $k_{3}$ 表示这种贡献，并设 $k_{3}$ 与 $\delta / D$ 成正比，其中 $\delta$ 为膜的厚度， $D$ 为膜的宽度，则

$$
k_{3}=\beta R \frac{\delta}{D},
$$

其中 $\beta$ 为常数, $R$ 为有效半径 $\left(\pi R^{2}=A\right)$.

为了减小 $k_{3}$, 应将 $\delta$ 取得尽量小, 但这又受到膜的强度的限制. 由几何关系及膜的受力分 析可得, 当 $R \gg D$, 有

$$
\frac{\delta}{D} \doteq \frac{p_{0}}{2 \sigma_{M}+p_{0}},
$$

其中 $\sigma_{M}$ 为橡胶材料的最大拉应力. 由此得

$$
k_{3} \doteq \beta \frac{p_{0} R}{2 \sigma_{M}+p_{0}}
$$

由理论上给出 $\frac{d A}{d x}$ 的准确表达式很困难, 但我们可以利用实验结果找到经验公式. 由 
$A=\pi R^{2}$, 得 $\frac{d A}{d x}=2 \pi R \frac{d R}{d x}$. 设 $\frac{d R}{d x}$ 与 $D$ 的 $n$ 次方成正比,并将(5)式右边后一项记作 $k_{2}$,得

$$
k_{2}=\alpha p_{0} R D^{n}
$$

其中 $\alpha 、 n$ 为常数. 由此,(5)式成为

$$
k_{0}=r\left(p_{0}+p_{a}\right) \frac{A_{0}^{2}}{V_{0}}+\alpha p_{0} R D^{n}+\beta \frac{p_{0} R}{2 \sigma_{M}+p_{0}} .
$$

我们利用实验结果中几组较合理的数据, 按照最小二乘法的原理,对 (10)式进行了拟合, 得到 了其中的几个常数: $n=2.853, \alpha=0.08631, \beta=6.670$. ( $k_{0}$ 的单位为 $\mathrm{N} / \mathrm{cm}, p_{0}$ 与 $p_{a}$ 为 10 $\mathrm{kPa}, A_{0}$ 为 $\mathrm{cm}^{2}, V_{0}$ 为 $\mathrm{cm}^{3}, R$ 为 $\mathrm{cm}, \sigma_{M}$ 为 $\left.10 \mathrm{kPa}\right)$. 于是得到这种空气弹簧的刚度半经验公式.

将 $R=\sqrt{A_{0} / \pi}, p_{0}=W / A_{0}$ 代人 (10)式,得

$$
k_{0}=r\left(\frac{W}{A_{0}}+p_{a}\right) \frac{A_{0}^{2}}{V_{0}}+\frac{a}{\sqrt{\pi}} D^{n} \frac{W}{A_{0}}+\frac{\beta}{\sqrt{\pi}} \frac{\sqrt{A_{0}}}{1+2 \sigma_{M} A_{0} / W},
$$

当 $W 、 V_{0} 、 D$ 确定之后，(11)式成为 $A_{0}$ 的一元函数. 显然, 这个函数有个极小值, 也就是说 $A_{0}$ 有个最佳取值.

针对我们目前的实验条件, 我们对 (11)式进行了优化计算. 取实验值 $W=2.43 \mathrm{kN}, \sigma_{M}=$ $0.883 \mathrm{MPa}$ ，当 $V_{0}=2.66 \times 10^{4} \mathrm{~cm}^{3}, D=1.0 \mathrm{~cm}$ 时， $A_{0 \mathrm{opt}}=53.0 \mathrm{~cm}^{2}, k_{0 \min }=30.1 \mathrm{~N} / \mathrm{cm}$, 自 振频率 $f=0.556 \mathrm{~Hz}$ ，这与实验所取的参数和结果相近. 当 $V_{0}=1.84 \times 10^{5} \mathrm{~cm}^{3}$ (加附加气 室后), $D=1.0 \mathrm{~cm}$ 时, $A_{0 \mathrm{opt}}=152 \mathrm{~cm}^{2}, k_{0 \mathrm{~min}}=18.0 \mathrm{~N} / \mathrm{cm}, f=0.43 \mathrm{~Hz}$. 实验上曾用这组 $V_{0} 、 D$ 值, 取 $A_{0}=50 \mathrm{~cm}^{2}$, 得到 $f=0.50 \mathrm{~Hz}$ 的结果. 按以上两组参数绘得的 (11) 式的函数 曲线如图 2 所示. 如果 $V_{0}=1.84 \times 10^{5} \mathrm{~cm}^{3}, D=0.6 \mathrm{~cm}$, 则算得 $A_{0 \mathrm{opt}}=95.8 \mathrm{~cm}^{2}, k_{0 \mathrm{~min}}=$ $10.0 \mathrm{~N} / \mathrm{cm}, f=0.32 \mathrm{~Hz}$. 以上结果表明, 这种空气弹簧可能达到的最低自振频率为 $0.3 \mathrm{~Hz}$ 。
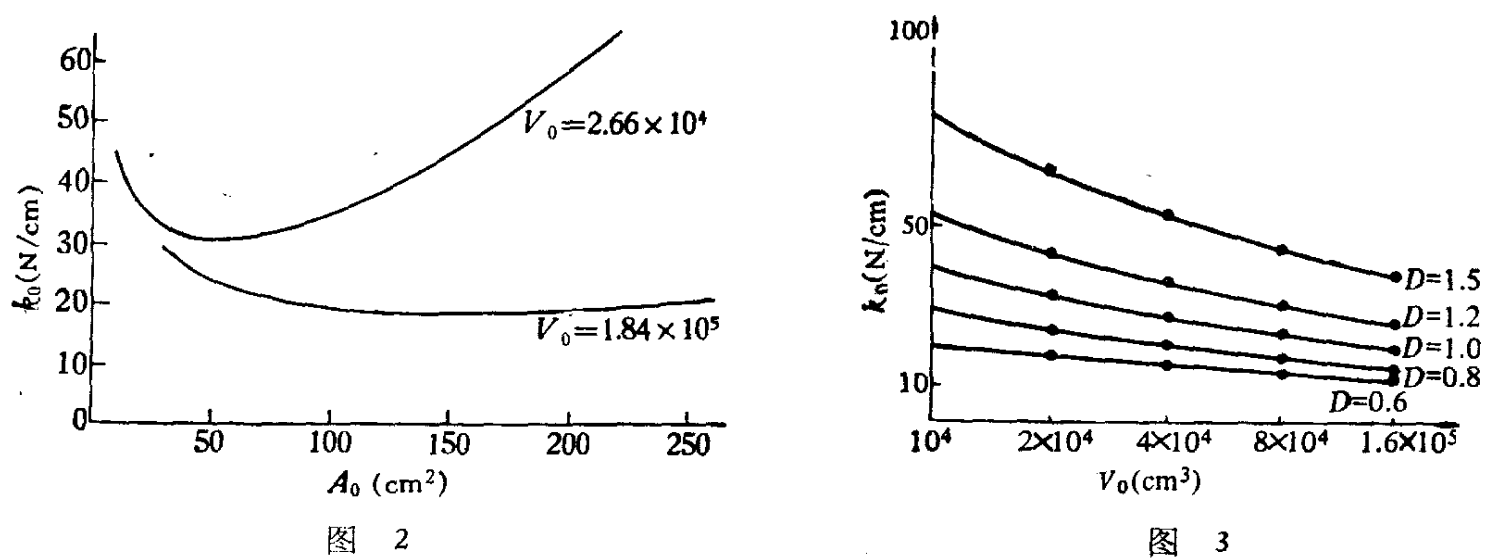

图 3 表示了 (11) 式中 $k_{1}$ 的最小值随 $V_{0}$ 和 $D$ 而变化的情况, 图中的圆点都是优化而得的 值. 计算时取 $W=2.45 \mathrm{kN}, \sigma_{M}=0.883 \mathrm{MPa}$ 。

\section{参考 文献}

[ 1 ] 徐步新、秦荣先, 物理学报, 31(1982), 1097 .

[2]阎永廉、秦荣先,物理学报,32(1983), 1586 .

[3] 阎永廉、秦柴先, 研究生院学报, 1(1984),2: 163.

[4] Fujimoto, M. K., Hirakawa, H., J. Phps. Soc. Japan, 46(1979), 703.

[5]张英会(主编)等,弹簧,第二十章,机械工业出版社, 北京, 1982,399. 